\title{
Preface to the special issue on computational methods in quantitative finance
}

\author{
Jun Sekine ${ }^{1}$
}

Published online: 19 October 2017

(C) The JJIAM Publishing Committee and Springer Japan KK 2017

The success of mathematical finance, which has revolutionized the financial world in the past 40 years, stands on the parallel development of efficient computational methods as well as more sophisticated mathematical models. For developing novel computational methods, an interdisciplinary approach involving a variety of methods from financial mathematics, stochastics, statistics, numerics, and scientific computing has been utilized.

This special issue focuses on the novel computational techniques in quantitative finance and analyses of related stochastic models. Eleven papers constitute this issue, for which Area 4 Editor Jun Sekine is responsible for the refereeing process. Those 11 are: "Implicit American Monte Carlo methods for nonlinear functional of future portfolio value" by Yusuke Morimoto; "FFT network for interest rate derivatives with Lévy processes" by Mei Choi Chiu, Zhuolu Xu, and Hoi Ying Wong; "Dual-curve Hull-White interest rate model with stochastic volatility" by Mei Choi Chiu, Wanyang Liang, and Hoi Ying Wong; "Approximation of eigenvalues of spot cross volatility matrix with a view toward principal component analysis" by Nien-Lin Liu and HoangLong Ngo; "Monte Carlo calibration to implied volatility surface under volatility models" by Chuan-Hsiang Han and Chien-Liang Kuo; "Optimal exercise boundary via intermediate function with jump risk" by Beom Jin Kim, Yong-Ki Ma, and Hi Jun Choe; "A dynamic programming approach to a consumption/investment and retirement choice problem under borrowing constraints" by Ho-Seok Lee, Byung Lim Koo, and Yong Hyun Shin; "Partial super-hedging of derivatives with model risk" by Koichi Matsumoto; "Towards the exact simulation using hyperbolic Brownian motion" by

Jun Sekine

sekine@sigmath.es.osaka-u.ac.jp

1 Division of Mathematical Science for Social Systems, Department of Systems Innovation,

Graduate School of Engineering Science, Osaka University, 1-3, Machikaneyama,

Toyonaka, Osaka 560-8531, Japan 
Yuuki Ida and Yuri Imamura; "On the difference between locally risk-minimizing and delta hedging strategies for exponential Lévy models" by Takuji Arai and Yuto Imai; and "On distributions of first passage times of martingales arising in some gambling problems" by Alexander Novikov and Shunsuke Kaji.

Several of the articles were written by the participants of the Fourth Asian Quantitative Finance Conference (AQFC), which was held at Osaka University's Nakanoshima Center February 21-23, 2016, in which we announced the plans for this special issue. We appreciate the efforts of the authors from the AQFC participants as well as those of the other authors in preparing their manuscripts. We are convinced that these 11 papers display various interesting aspects of "computational finance", which is a diverging, interdisciplinary research area of applied mathematics. 